ORIGINAL

\title{
COMPARACIÓN ENTRE VARIAS POBLACIONES ESTÁNDAR PARA EL AJUSTE POR EDADES
}

\author{
Javier Llorca Díaz (1), Dolores Prieto Salceda (1), Trinidad Dierssen Sotos (1), Miguel Delgado- \\ Rodríguez (1,2)
}

(1) Cátedra de Medicina Preventiva y Salud Pública. Facultad de Medicina. Universidad de Cantabria.

(2) Cátedra de Medicina Preventiva y Salud Pública. Universidad de Jaén.

\section{RESUMEN}

Fundamento: Analizar el efecto que tiene la elección de una población de referencia sobre diferentes indicadores derivados de las tasas de mortalidad ajustadas por edad.

Métodos: Las tasas de mortalidad por diferentes causas de muerte en España de 1971 a 1992 son ajustadas empleando cuatro poblaciones de referencia: poblaciones españolas de 1971 y 1992, población estándar europea y población estándar mundial. Los resultados obtenidos con las cuatro poblaciones se comparan empleando tres indicadores: diferencia entre las tasas de 1992 y 1971 , razón entre las tasas de 1992 y 1971 y cambio anual porcentual entre 1971 y 1992.

Resultados: En la mayor parte de las causas de muerte estudiadas incluyendo el total de causas, la cardiopatía isquémica y casi todos los tumores, la razón de tasas y el porcentaje de cambio anual son similares con independencia de cuál sea la población estándar empleada. En cambio, la diferencia de tasas es muy diferente en función de la población de referencia. En las enfermedades infecciosas y el cáncer de testículo se produce la situación contraria: la diferencia de tasas es robusta mientras que la razón de tasas varía con la población estándar. Finalmente, la mortalidad por neumonía, enfermedad de Parkinson y cáncer de encéfalo muestra cambios en los tres indicadores utilizados.

Conclusiones: Es necesario realizar un análisis de las tasas especificas por edad antes de proceder a su ajuste. Este análisis permite averiguar si el ajuste por edades es correcto y qué indicador (diferencia, razón o cambio porcentual) será adecuado para realizar comparaciones.

Palabras clave: Métodos epidemiológicos. Mortalidad. Estandarización.

Correspondencia:

Dr. Javier Llorca Díaz

Cátedra de Medicina Preventiva y Salud Pública

Facultad de Medicina de la Universidad de Cantabria

Avda. Cardenal Herrera Oria s/n

39011 Santander

Teléfono: 942201993

Fax: 942201903

Correo electrónico: 1lorcaj@medi.unican.es.
ABSTRACT

\section{Comparison betwen Several Standard Populations for Agc-adjusting}

Background: To analyse the effect the choice of reference population has on different indicators derived from mortality rates age-adjusteing.

Methods: The mortality rates for different causes of death in Spain from 1971 to 1992 were adjusted by using four reference populations: Spanish populations from 1971 and 1992, the Standard European population and the standard world population. The results obtained with the four populations were compared by applying three indicators: difference between the rates of 1992 and 1971 , ratio between the rates of 1992 and 1971 and annual percentage change between 1971 and 1992.

Results: In the majority of the causes of death studied, including the total, ischemic heart disease and almost all of the tumours, the ratio between the rates and the percentage of annual change are similar regardless of the standard population used. However, the difference in ratcs is very noticcable in relation to the reference population. In the infectious diseases and testis cancer, the opposite occurs: the different in rates is steadfast while the ratio between the rates varies with the standard population. Finally, the mortality due to pneumonia, Parkinson's disease and encephalic cancer show changes all three indicators used.

Conclusions: An analysis of the specific rates by age must be made before proceeding with their adjustment. This analysis will enable us to ascertain whether the adjustment by age is correct and which indicator (difference, ratio or percentage change) will be appropriate for making comparisons.

Key Words: Epidemiological Methods. Mortality. Standardisation. 


\section{INTRODUCCIÓN}

El ajuste por edades es una técnica ampliamente empleada en epidemiología para eliminar el sesgo de confusión introducido por la diferente composición por edad de varias poblaciones y para presentar de forma resumida los datos procedentes de tasas específicas por edades ${ }^{1}$. El método más empleado, conocido como ajuste directo, fue propuesto por $\mathrm{Neison}^{2}$ en 1844 y consiste en calcular la media ponderada de las tasas específicas por edad, empleando como pesos la composición de una población de referencia arbitraria.

Es sabido que las tasas ajustadas sólo pueden compararse entre sí cuando el ajuste se ha producido respecto de la misma población de referencia; es decir, saber que la mortalidad por cardiopatía isquémica ajustada respecto de la población española de 1971 era 82 por 100.000 en 1980 y ajustada respecto de la población española de 1991 era 127 por 100.000 en 1975 , no permite deducir que se ha producido un fuerte descenso en la mortalidad por cardiopatía isquémica entre 1975 y 1980.

Probablemente es menos conocido que la elección de la población de referencia puede alterar la tendencia de las tasas ajustadas. Por ejemplo, un estudio sobre la mortalidad por cáncer en Estados Unidos indicó que entre 1980 y 1988 la mortalidad ajustada por edad había disminuido un $0,1 \%$ si se empleaba como referencia la población norteamericana de 1940 , había aumentado un $1,5 \%$ si se usaba la población de 1980 y había aumentado un 2,5\% si la referencia era la población norteamericana proyectada para el año $2050^{3}$.

Un reciente artículo de Choi et al analiza las condiciones en que puede emplearse el ajuste por edades y la interpretación de sus resultados ${ }^{4}$. De manera resumida, se consideran tres situaciones: (1) las tasas específicas por edad permanecen constantes, (2) la diferencia de las tasas específicas por edad permanece constante y (3) la razón entre las tasas específicas por edad permanece constante. En el primer caso, tanto la diferencia como la razón entre las tasas ajustadas permanecen constantes cualquiera que sea la población de referencia elegida; en el segundo escenario, la diferencia entre las tasas ajustadas permanece constante, pero la razón entre las tasas ajustadas puede variar en función de la población de referencia. En el tercer caso, la razón entre las tasas ajustadas permanece constante pero la diferencia entre las tasas ajustadas varía con la población de referencia.

El objetivo de este artículo es comprobar el efecto que tiene la elección de la población de referencia sobre la mortalidad ajustada por edad en España, considerando un abanico amplio de causas de muerte.

\section{MATERIAL Y MÉTODO}

El número de muertes por sexo, edad (grupos quinquenales) y por causa desde 1971 a 1992 se han obtenido de las publicaciones del Movimiento Natural de la Población Española ${ }^{5}$. Se han considerado las siguientes causas de muerte (todas referidas a varones, excepto el cáncer de mama y el cáncer de cervix): todas las causas (CIE-8: 001-999, CIE-9: 001-999), enfermedades infecciosas (CIE-8: 001-136, CIE-9: 001-139), tuberculosis (CIE-8: 010-019, CIE-9: 010-018), cáncer de estómago (CIE-8: 151, CIE-9: 151), cáncer de colon y recto (CIE-8: 153-154, CIE-9: 153-154), cáncer de páncreas (CIE-8: 157, CIE-9: 157), cáncer de pulmón (CIE-8: 162, CIE-9: 162), cáncer de mama en la mujer (CIE-8: 174, CIE-9: 174), cáncer de cérvix (CIE-8: 180, CIE-9: 180), cáncer de próstata (CIE-8: 185, CIE-9: 185), cáncer de testículo (CIE-8: 186, CIE-9: 186), cáncer de encéfalo (CIE-8: 191, CIE-9: 191), enfermedad de Parkinson (CIE-8: 342, CIE-9: 332), enfermedad de la motoneurona (CIE-8: 348, CIE-9: 335.2), esclerosis múltiple (CIE-8: 340, CIE-9: 340), cardiopatía isquémica (CIE-8: 410-414, CIE-9: 410-414), neumonía (CIE-8: 480-486, CIE-9: 480-487), bronquitis crónica y enfisema (CIE8: 491-492, CIE-9: 491-492), y caídas accidentales (CIE-8: E880-E887, CIE-9: E880-E888). Estas causas se han elegido para mostrar el comportamiento de causas de muerte muy variadlas.

Las poblaciones de cada grupo de edad y sexo a mitad de cada año se han calculado por 
interpolación exponencial a partir de las poblaciones censales de 1970, 1981 y 1991.

La tasa de mortalidad de cada causa y año se ha ajustado por edad empleando cuatro poblaciones diferentes como referencia: población española de $1971^{6}$, población española de $1991^{7}$, población estándar mundial8 y población estándar europea ${ }^{8}$ (tabla 1). En líneas generales, la población estándar mundial y la población de española de 1971 muestran una distribución más joven que la estándar europea y la española de 1991.

Para cada combinación de causa de muerte y población de referencia se han calculado tres parámetros: la diferencia entre la tasa ajustada en 1992 y la tasa ajustada en 1971, la razón entre la tasa ajustada en 1992 y la tasa ajustada en 1971, y el porcentaje de variación anual (estimado por interpolación exponencial) entre 1971 y 1992.

\section{RESULTADOS}

Los principales resultados se muestran en la tabla 2. En la mayoría de las causas de muerte, la diferencia entre tasas ajustadas es muy sensible a la elección de la población de referencia, mientras que la razón entre las tasas y el porcentaje anual de cambio muestran pocos cambios en función de la población elegida. Por ejemplo, considerando todas las causas de muerte se encuentra que con la población estándar mundial se ha producido un descenso de 201 muertes por 100.000 personas entre 1971 y 1992 , mientras que si ajustamos por la población española de 1991 el descenso es de 366 muertes por 100.000. En cambio, el porcentaje de descenso anual ha sido del $1,6 \%$ y $1,5 \%$, respectivamente. En la figura 1 se presenta la evolución del índice de mortalidad relativo desde 1971 hasta 1992, obtenido dividiendo la mortalidad ajustada de cada año

Tabla 1

Poblaciones de referencia

\begin{tabular}{|c|c|c|c|c|c|c|c|c|}
\hline \multirow[b]{2}{*}{$\begin{array}{l}\text { Grupo de } \\
\quad \text { edad }\end{array}$} & \multicolumn{4}{|c|}{ Varones } & \multicolumn{4}{|c|}{ Mujeres } \\
\hline & $\begin{array}{c}\text { España } \\
1971\end{array}$ & $\begin{array}{c}\text { España } \\
1991\end{array}$ & $\begin{array}{l}\text { Estándar } \\
\text { mundial }\end{array}$ & $\begin{array}{l}\text { Estándar } \\
\text { europeo }\end{array}$ & $\begin{array}{c}\text { España } \\
1971\end{array}$ & $\begin{array}{c}\text { España } \\
1991\end{array}$ & $\begin{array}{l}\text { Estándar } \\
\text { mundial }\end{array}$ & $\begin{array}{l}\text { Estándar } \\
\text { europeo }\end{array}$ \\
\hline $0-4$ & 1656089 & 1070197 & 12071 & 6648 & 1575140 & 996632 & 11702 & 6018 \\
\hline $5-9$ & 1628363 & 1231378 & 10824 & 6800 & 1550817 & 1146508 & 10472 & 6160 \\
\hline $10-14$ & 1559435 & 1539255 & 9954 & 7108 & 1501467 & 1451777 & 9609 & 6452 \\
\hline $15-19$ & 1370774 & 1686846 & 9989 & 7570 & 1330437 & 1594414 & 9627 & 6863 \\
\hline $20-24$ & 1278599 & 1677902 & 9477 & 8163 & 1259547 & 1602074 & 9137 & 7438 \\
\hline $25-29$ & 1150145 & 1649431 & 8458 & 8206 & 1154566 & 1599617 & 8204 & 7552 \\
\hline $30-34$ & 1023904 & 1470356 & 7355 & 7811 & 1034458 & 1459711 & 7175 & 7258 \\
\hline $35-39$ & 1167847 & 1277248 & 6585 & 7448 & 1201158 & 1268837 & 6476 & 6986 \\
\hline $40-44$ & 1137407 & 1214278 & 5326 & 7068 & 1159433 & 1220413 & 5253 & 6661 \\
\hline $45-49$ & 1047941 & 1110150 & 4341 & 5997 & 1088622 & 1124219 & 4335 & 5739 \\
\hline $50-54$ & 837588 & 966303 & 3994 & 5937 & 927358 & 1006894 & 4061 & 5817 \\
\hline $55-59$ & 743699 & 1074506 & 3486 & 5521 & 869284 & 1150742 & 3604 & 5585 \\
\hline $60-64$ & 688303 & 992553 & 2912 & 5015 & 816686 & 1103280 & 3179 & 5463 \\
\hline $65-69$ & 566967 & 827679 & 2167 & 4139 & 713865 & 978507 & 2591 & 5196 \\
\hline $70-74$ & 384159 & 572181 & 1424 & 2449 & 528189 & 780612 & 1837 & 3392 \\
\hline $75-79$ & 236065 & 403467 & 958 & 2228 & 364756 & 635777 & 1406 & 3536 \\
\hline $80-84$ & 120735 & 250034 & 429 & 1094 & 210543 & 444383 & 814 & 2076 \\
\hline 85 y más & 59121 & 142426 & 250 & 798 & 124022 & 304311 & 518 & 1808 \\
\hline
\end{tabular}


Tabla 2

Comparación entre las tablas ajustadas de 1992 y 1971

\begin{tabular}{|c|c|c|c|c|c|}
\hline \multirow[b]{2}{*}{ Causa de muerte } & & \multicolumn{4}{|c|}{ Población de referencia } \\
\hline & & España-1971 & España-199I & Estándar mundial & Estândar europeo \\
\hline \multirow[t]{3}{*}{ Todas las causas } & Diferencia & $-279,44$ & $-366,30$ & $-200,96$ & $-354,34$ \\
\hline & Razón & 0,70 & 0,71 & 0,71 & 0,71 \\
\hline & Porcentaje anual & $-1,6$ & $-1,5$ & $-1,6$ & -1.5 \\
\hline \multirow[t]{3}{*}{ Cáncer } & Diferencia & 35,09 & 48,46 & 23,26 & 47,17 \\
\hline & Razón & 1,22 & 1,23 & 1,21 & 1,24 \\
\hline & Porcentaje anual & 0,9 & 1,0 & 0,9 & 1,0 \\
\hline \multirow[t]{3}{*}{ Ca. de pulmón } & Diferencia & 27,34 & 35,94 & 18,37 & 34,71 \\
\hline & Razón & 2,03 & 2,06 & 2,02 & 2,06 \\
\hline & Porcentaje anual & 3,3 & 3,3 & 3,2 & 3,3 \\
\hline \multirow[t]{3}{*}{ Ca. de testículo } & Diferencia & 0,09 & 0,13 & 0,11 & 0,11 \\
\hline & Razón & 1,47 & 1,59 & 1,65 & 1,59 \\
\hline & Porcentaje anual & 1,8 & 2,1 & 2,3 & 1,9 \\
\hline \multirow[t]{3}{*}{ Ca. próstata } & Diferencia & 0,16 & 0,63 & 0,11 & 0,77 \\
\hline & Razón & 1,01 & 1,03 & 1,01 & 1,04 \\
\hline & Porcentaje anual & 0,1 & 0,2 & 0,1 & 0,2 \\
\hline \multirow[t]{3}{*}{ Ca. encéfalo } & Diferencia & $-0,37$ & $-0,19$ & $-0,50$ & $-0,29$ \\
\hline & Razón & 0,92 & 0,96 & 0,86 & 0,94 \\
\hline & Porcentaje anual & $-0,4$ & $-0,2$ & $-0,7$ & $-0,3$ \\
\hline \multirow[t]{3}{*}{ Ca. màma } & Diferencia & 8,20 & 10,60 & 5,67 & 10,87 \\
\hline & Razón & 1,57 & 1,62 & 1,56 & 1,62 \\
\hline & Porcentaje anual & 2,1 & 2,2 & 2,0 & 2,2 \\
\hline \multirow[t]{3}{*}{ Ca. cérvix } & Diferencia & 1,18 & 1,41 & 0,86 & 1,44 \\
\hline & Razón & 2,34 & 2,34 & 2,37 & 2,33 \\
\hline & Porcentaje anual & 3,9 & 3,9 & 4,0 & 3,9 \\
\hline \multirow[t]{3}{*}{ Ca. páncreas } & Diferencia & 2,86 & 3,77 & 1,95 & 3,69 \\
\hline & Razón & 1,79 & 1,80 & 1,77 & 1,81 \\
\hline & Porcentaje anual & 2,7 & 2,7 & 2,6 & 2,7 \\
\hline \multirow[t]{3}{*}{ Ca. colorrectal } & Diferencia & 5,57 & 7,85 & 3,70 & 7,58 \\
\hline & Razón & 1,50 & 1,52 & 1,48 & 1,52 \\
\hline & Porcentaje anual & 1,9 & 1,9 & 1,8 & 1,9 \\
\hline \multirow[t]{3}{*}{ Ca. estómago } & Diferencia & $-16,53$ & $-21,94$ & $-11,21$ & $-21,04$ \\
\hline & Razón & 0,47 & 0,48 & 0,47 & 0,48 \\
\hline & Porcentaje anual & $-3,3$ & $-3,3$ & $-3,3$ & $-3,3$ \\
\hline \multirow[t]{3}{*}{ Card. isquémica } & Diferencia & 2,10 & 4,16 & 1,27 & 4,10 \\
\hline & Razón & 1,03 & 1,04 & 1,03 & 1,04 \\
\hline & Porcentaje anual & 0,3 & 0,4 & 0,3 & 0,4 \\
\hline
\end{tabular}


Tabla 2 (continuación)

Comparación entre las tablas ajustadas de 1992 y 1971

\begin{tabular}{|c|c|c|c|c|c|}
\hline \multirow[b]{2}{*}{ Causa de muerte } & & \multicolumn{4}{|c|}{ Población de referencia } \\
\hline & & España-1971 & España-1991 & Estândar mundial & Estándar europeo \\
\hline \multirow[t]{3}{*}{ BOC y enfisema } & Diferencia & $-26,72$ & $-38,70$ & $-17,78$ & $-36,95$ \\
\hline & Razón & 0,18 & 0,19 & 0,18 & 0,19 \\
\hline & Porcentaje anual & $-7,5$ & $-7,3$ & $-7,5$ & $-7,3$ \\
\hline \multirow[t]{3}{*}{ Enf. infecciosas } & Diferencia & $-19,91$ & $-18,94$ & $-17,97$ & $-19,63$ \\
\hline & Razón & 0,31 & 0,38 & 0,28 & 0,37 \\
\hline & Porcentaje anual & $-5,2$ & $-4,3$ & $-5,6$ & $-4,4$ \\
\hline \multirow[t]{3}{*}{ Neumonia } & Diferencia & $-29,04$ & $-34,94$ & $-23,88$ & $-34,50$ \\
\hline & Razón & 0,27 & 0,33 & 0,24 & 0,32 \\
\hline & Porcentaje anual & $-5,7$ & $-5,0$ & $-6,2$ & $-5,0$ \\
\hline \multirow[t]{3}{*}{ Tuberculosis } & Diferencia & $-13,64$ & $-16,03$ & $-10,09$ & $-16,02$ \\
\hline & Razón & 0,14 & 0,15 & 0,15 & 0,15 \\
\hline & Porcentaje anual & $-8,50$ & $-8,3$ & $-8,4$ & $-8,3$ \\
\hline \multirow[t]{3}{*}{ Enf motoneurona } & Diferencia & 0,43 & 0,52 & 0,30 & 0,50 \\
\hline & Razón & 1,84 & 1,83 & 1,81 & 1,82 \\
\hline & Porcentaje anual & 2,8 & 2,8 & 2,7 & 2,8 \\
\hline \multirow[t]{3}{*}{ Enf. Parkinson } & Diferencia & $-1,06$ & $-1,24$ & $-0,71$ & $-1,16$ \\
\hline & Razón & 0,63 & 0,70 & 0,62 & 0,70 \\
\hline & Porcentaje anual & $-2,1$ & $-1,6$ & $-2,1$ & $-1,6$ \\
\hline \multirow[t]{3}{*}{ Escl. múltiple } & Diferencia & $-0,28$ & $-0,39$ & $-0,19$ & $-0,39$ \\
\hline & Razón & 0,52 & 0,47 & 0,54 & 0,47 \\
\hline & Porcentaje anual & $-3,0$ & $-3,3$ & $-2,8$ & $-3,3$ \\
\hline \multirow[t]{3}{*}{ Caídas accident. } & Diferencia & $-4,87$ & $-5,95$ & $-4,34$ & $-5,87$ \\
\hline & Razón & 0,36 & 0,36 & 0,35 & 0,36 \\
\hline & Porcentaje anual & $-4,6$ & $-4,5$ & $-4,7$ & $-4,5$ \\
\hline
\end{tabular}

Diferencia: diferencia (en tasas de mortalidad por 100.000) entre las tasas ajustadas por edad en 1992 y las tasas ajustadas por edad en 1971. Razón: razón entre las tasas ajustadas por edad en 1992 y las tasas ajustadas por edad en 1971.

Porcentaje anual: porcentaje de incremento anual entre 1971 y 1992, estimado asumiendo un crecimiento exponencial.

entre la de 1971. El resultado son cuatro líneas (una para cada población de referencia) que se presentan en escala logarítmica (adecuada para observar los cambios en el valor relativo). Puede observarse que estas líneas no divergen, lo que indica que el cambio porcentual es similar respecto a cualquiera de las poblaciones de referencia. En la figura 2 se presenta la evolución de la diferencia de tasas desde 1971 hasta 1992, obtenida restando la mortalidad ajustada de 1971 de la correspondiente a cada año. Las cuatro líneas obtenidas (una para cada población de referencia) se presentan en escala aritmética (adecuada para observar cambios en el valor absoluto). Puede observarse una clara divergencia entre estas cuatro líneas, indicando que el cambio en la diferencia de tasas depende de la población de referencia elegida. Una situación similar (es decir, una diferencia de tasas muy variable, dependiendo de la población estándar, pero una razón de tasas y un porcentaje anual de cambio muy estables) 
se encuentra en el total de muertes por cáncer, la mayoría de los cánceres, la cardiopatía isquémica, la broncopatía crónica, la tuberculosis y la enfermedad de la motoneurona.

En las enfermedades infecciosas, en cambio, se encuentra que la diferencia entre las tasas ajustadas de 1971 y de 1992 es muy similar cualquiera que sea la población de referencia elegida. Sin embargo, hay gran variabilidad en la razón de tasas ajustadas (mínimo 0,28 con la población estándar mundial, máximo 0,38 con la población española de 1991) y en el porcentaje de variación anual (descenso de $5,6 \%$ anual con la población estándar mundial y de $4,3 \%$ anual con la población española de 1992). Las figuras 3 y 4 muestran la evolución del índice de mortalidad relativa y de la diferencia de tasas respectivamente (ambas figuras se han construido de manera similar a las figuras 1 y 2). En la figura 3 puede verse que las líneas divergen, mostrando que el cambio porcentual es diferente según la población estándar empleada. En cambio, en la figura 4 las líneas no divergen, indicando que el cambio en valor absoluto es similar con cualquier población de referencia. Debe llamarse la atención sobre una aparente paradoja: la población que menor diferencia de tasas ajustadas ofrece es también la que indica un mayor cambio porcentual anual. Una situación parecida, aunque menos llamativa, se produce en el cáncer de testículo.

Un tercer patrón de cambios se presenta en la mortalidad por neumonía. Ninguno de los tres indicadores empleados (la diferencia de tasas ajustadas, la razón de tasas ajustadas y el porcentaje anual de cambio en las tasas ajusta-

Figura 1

Evolución del índice de mortalidad relativa desde 1971 hasta 1992 empleando las cuatro poblaciones estándar (todas las causas). Se ha obtenido dividiendo la mortalidad ajustada correspondiente a cada año entre la de 1971. Cada línea representa una población de referencia.

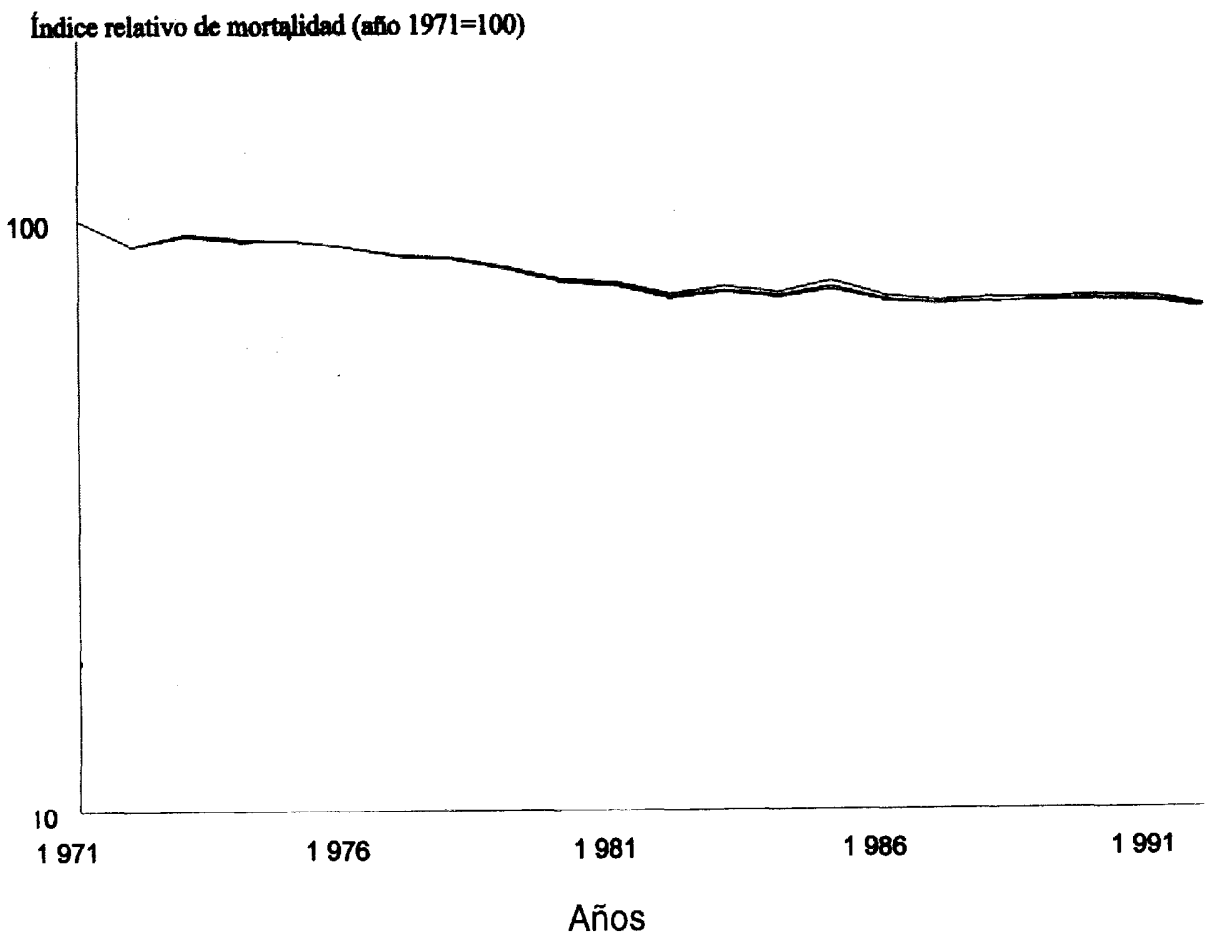


Figura 2

Evolución de la diferencia de tasas de mortalidad desde 1971 hasta 1992 emplcando las cuatro poblaciones estándar (todas las causas). Se ha obtenido restando la mortalidad ajustada correspondiente a cada año de la de 1971. Cada línea representa una población de referencia.

Jiterencia de mortalidaa en tasa pui iwiut
atho $1971=0$ )

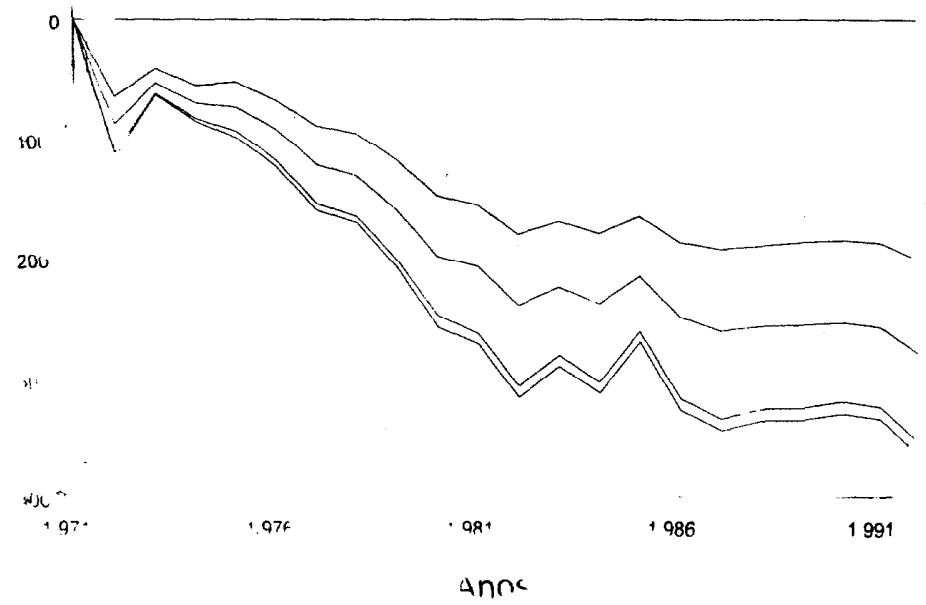

Figura 3

Evolución del índice de mortalidad relativa desde 1971 hasta 1992 empleando las cuatro poblaciones estándar (enfermedades infecciosas). Se ha obtenido dividiendo la mortalidad ajustada correspondiente a cada año entre la de 1971. Cada línea representa una población de referencia. Se ha mantenido la misma escala que en la figura 1 para facilitar la comparación.

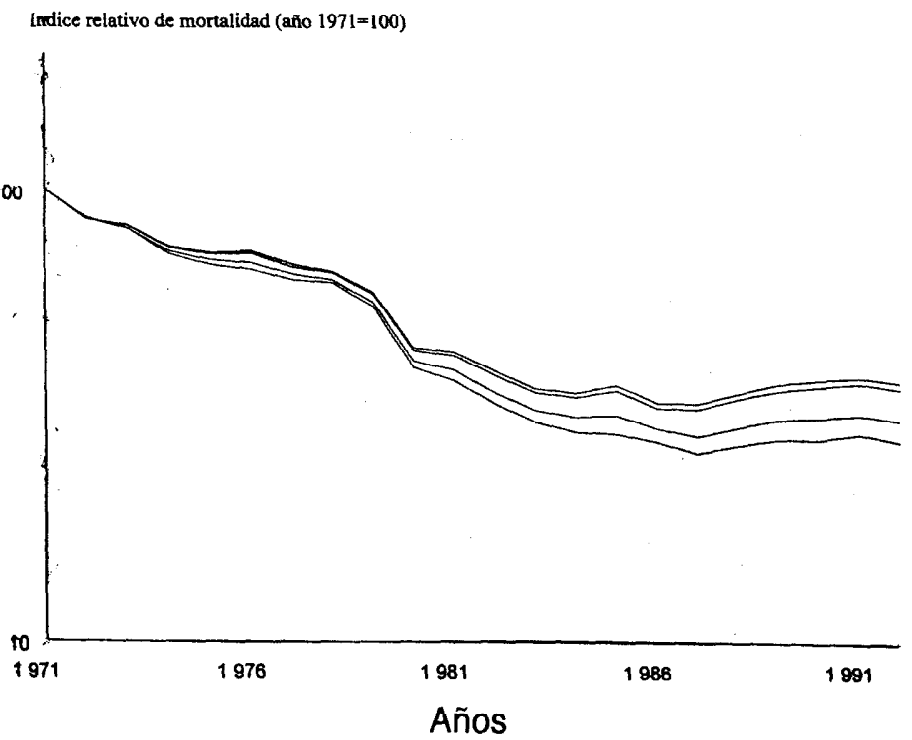


das) presenta resultados similares con las cuatro poblaciones estándar. Así el máximo cambio en la diferencia de tasas ocurre con la población española de $1991(-34,95$ por 100000$)$, en la que también ocurre el menor cambio porcentual $(-5,0 \%$ al año). Aunque no se muestra una figura, este patrón corresponde a tendencias divergentes tanto en escala aritmética como en escala logarítmica. La misma falta de reproducibilidad de todos los indicadores se da en la enfermedad de Parkinson y en el cáncer de encéfalo.

En líneas generales, la población estándar europea y la población española de 1991 ofrecen resultados muy similares en los tres indicadores empleados. La población española de 1971 y, más aún, la población estándar mundial suelen presentar diferencias de tasas menores que las anteriores.

\section{Figura 4}

Evolución de la difcrencia de tasas de mortalidad desde 1971 hasta 1992 empleando las cuatro poblaciones estándar (enfermedades infecciosas). Se ha obtenido restando la mortalidad ajustada correspondiente a cada año de la de 1971. Cada línea representa una población de referencia. Se ha reducido la escala del eje Y respecto de la figura 2 para facilitar la visualización; de mantenerse la misma escala que en la figura 2 las diferencias entre las cuatro líneas serían aún menores.

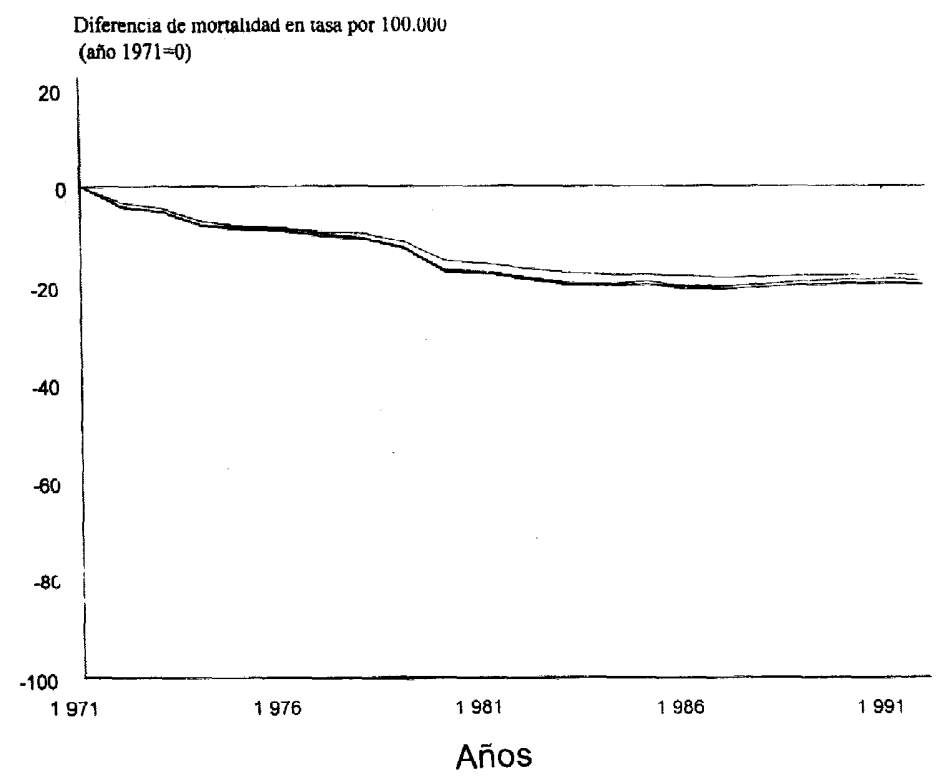

\section{DISCUSIÓN}

La comparación de las tasas de mortalidad entre diferentes poblaciones suele realizarse después de un ajuste por edad. Esta técnica es sencilla y útil pero requiere una visión crítica sobre las condiciones en las que se realiza. Las inferencias que se realizan después de comparar tasas ajustadas por edad requieren, para ser válidas, que las tasas específicas por edad tengan una relación consistente; es decir, que se muevan en la misma dirección y con la misma magnitud relativa ${ }^{1,3}$. La condición «que se muevan en la misma dirección» es operativamente fácil de comprobar: consiste en observar en el análisis estratificado que todas las tasas específicas por edad aumentan (o disminuyen) en el periodo considerado. La segunda condición («con la misma magnitud relativa») es conceptualmente más difícil porque depende de la medida empleada para la comparación. Si las tasas específicas por edad muestran un cambio similar en valor absoluto, entonces 
el ajuste de tasas es una buena medida para realizar inferencias sobre la diferencia en las tasas ajustadas; en estas condiciones, la diferencia de tasas no se verá afectada por la elección de la población estándar. En cambio, si las tasas específicas por edad muestran un cambio similar en valor relativo, entonces el ajuste de tasas sólo puede ser empleado de manera consistente para analizar la razón de tasas ajustadas y el cambio porcentual. Por último, si las tasas específicas por edad muestran cambios que no son similares ni en valor absoluto ni en valor relativo, entonces el ajuste de tasas no debe ser empleado porque sus resultados pueden ser tan arbitrarios como la elección de la población.

Los resultados de este trabajo están limitados por la utilización de datos procedentes de dos revisiones de la Clasificación Internacional de Enfermedades ( $8^{\mathrm{a}}$ y $9^{\mathrm{a}}$ revisión) lo que puede originar que parte de la evolución de las tasa de mortalidad se deba a cambios en la definición de la enfermedad. Estos resultados muestran que el ajuste de tasas por edad es un procedimiento robusto (esto es, no afectado por la elección de la población de referencia) en la mayoría de las causas de muerte sólo si se va a emplcar para analizar la razón de tasas o el porcentaje de cambio. En algunas enfermedades, fundamentalmente infecciosas pero también en algunas degenerativas, el ajuste de tasas sólo puede emplearse de manera consistente en el análisis de la diferencia de tasas pero no en el de la razón de tasas.

Los dos párrafos anteriores tienen consecuencias importantes en epidemiología: la inconsistencia del ajuste de tasas en la diferencia de riesgos pucde hacer que las inferencias sobre riesgos atribuibles resulten erróneas. En las pocas enfermedades en las que el ajuste de tasas es inconsistente para la razón de tasas, las inferencias sobre riesgo relativo y fracciones etiológicas pueden ser equivocadas. Como consecuencia, en los trabajos científicos en los que se realice un ajuste de tasas, debe indicarse cuál ha sido la población de referencia indicada, no sólo para garantizar la validez externa (es decir: la comparabilidad con otros estudios procedentes de otras poblaciones) sino también para garantizar la validez interna (es decir: para que cl lector pueda interpretar el verdadero significado de las diferencias o razones de tasas ajustadas).

La coincidencia de resultados entre la población estándar europea y la población española de 1991 es consecuencia de que se trata de poblaciones de composición muy similar (tabla 1), mientras que la población estándar mundial y la española de 1971 tienen una composición claramente más joven.

En conclusión, el ajuste de tasas por edad debe realizarse después de estudiar las tasas específicas por edad. De esta forma puede conocerse si el ajuste puede llevarse a cabo de manera correcta y qué indicador de cambio en las tasas ajustadas resultará adecuado en ese caso concreto. Este es el método correcto para evitar que la elección, necesariamente arbitraria, de la población de referencia convierta en arbitrarios los resultados e invalide las inferencias que se realicen a partir de ellos.

\section{BIBLIOGRAFÍA}

1. Curtin LR, Klein RJ. Direct standardization (age-adjusted death rates). En: Healthy People 2000 statistical notes. $N^{\circ} 6$. Atlanta: Centers for Disease Control and Prevention; 1995.

2. Neison FGP. On a method recently proposed for conducting inquiries into the comparative sanitary condition of various districts. Lond $\mathrm{J}$ R Stat Soc 1844; 7:40-48. Cited in: Feinleib M. A reconsideration of age adjustment. En: National Center for Health Statistics. Reconsidering age-adgustment procedures: workshop proceedings. Atlanta: Centers for Disease Control and Prevention, 1992:4. (Vital and health statistics, Series 4: Documents and committee reports, núm 29) (DHSS publication núm (PHS) 0276-4733).

3. Kleinmann JC. The use of multiple standards. En: National Center for Health Statistics. Reconsidering age-adgustment procedures: workshop proceedings. Atlanta: Centers for Disease Control and Prevention, 1992:4. (Vital and health statistics, Series 4: Documents and committee reports, núm 29) (DHSS publication núm (PHS) 0276-4733). 
4. Choi BCK, Guia NA, Walsh P. Look before you leap: stratifay before you standardize. Am J Epidemiol 1999; 149:1087-96.

5. Instituto Nacional de Estadística. Movimicnto Natural de la Población. Tomo III: Defunciones según la causa de muerte (años 1971 a 1992). Madrid: INE Artes Gráficas; 19731994.
6. INE. Evolución de la Población Española en el período 1961-1978. Madrid: INE Artes Gráficas;1980.

7. INE. Anuario Estadístico de España Año 1993. Madrid: INE Artes Gráficas; 1994.

8. United Nations. World Population Prospects 1990. New York: United Nations; 1991. 